\title{
Non-differential conditioning of operant responding across a sound intensity continuum'
}

\author{
Frederiek W. Hegge, Rosemary Pierrel, J. Gilmour Sherman and Stephen Sadowsky 2 \\ BROWN UNIVERSITY
}

\begin{abstract}
Four groups of rats were conditioned to bar-piress in the presence of either nine sound intensities, a single intensity, or no imposed sound field. The distribution of responding and the range of observed response rate are similar under these three conditions. No significant facilitative, suppressive or preferential effect upon responding was observed.
\end{abstract}

\section{Problem}

The present study examines possible differential effects upon operant responding of a range of sound intensities unrelated to the reinforcement program. Hull (1949), in discussing stimulus intensity dynamism, (V), suggested that, when dealing with intensive continua, response strength will be a function of the intensity of the stimulus. Other experiments have pointed to the aversive properties of high intensity acoustic stimuli (Barry \& Harrison, 1957; Azrin, 1958; Harrison \& Abelson, 1959; Campbell, 1955), whereas Blough (1959) has noted preferential responding to mid-range intensities along a light intensity continuum.

The present study used a range of stimuli identical with that being employed in our laboratory in a series of studies dealing with various parameters of discrimination and generalization. It seemed important to determine whether or not these intensities, per se, were effective in producing facilitation, suppression or preference in responding, since evidence of discrimination would have to be assessed in terms of any such baseline effects.

\section{Method}

Ss were 16 Sprague-Dawley rats between 90-110 days old. The Ss were reduced to $80 \%$ of their ad libitum fed weights and so maintained. Sessions were run daily at the same hour; four Ss were run simultaneously. Sound-shielded enclosures equipped with speaker, retractable bar, water bottle, pellet dispenser and pellet dish were used. The programming of sound stimuli, reinforcement and recording of responses was fully automated and controlled from an adjacent room. The sound intensities presented were all $4 \mathrm{~K}$ cps tones. The reference intensity was $100 \mathrm{db}$ above .0002 dyne per $\mathrm{cm}^{2}$. The other eight intensities encompassed a range of $40 \mathrm{db}$ attenuation from this value in steps of $5 \mathrm{db}$. Whenever there was a change from one intensity level to another, a "fader" amplifier reduced the signal to zero amplitude and then returned it to the new amplitude. These $0.5 \mathrm{sec}$. fades eliminated switching transients. A more detailed account of the equipment used is given in Pierrel \& Sherman (1960).
The Ss were divided into four groups of four Ss each. Each $\mathrm{S}$ was exposed to a daily session of $2 \mathrm{hr}$. and $6 \mathrm{~min}$. Groups I and II were exposed to identical treatments. For these two groups all nine sound intensities were presented in a counterbalanced "random" order. The range of intensity presentations was from 1 to $3 \mathrm{~min}$. Each intensity was presented for a total of $14 \mathrm{~min}$. per session. Reinforcement was programmed on a VI $1 \mathrm{~min}$. schedule that was carefully constructed to ensure equal potential reinforcement at each intensity.

Groups III and IV were run as controls; they were not exposed to the nine intensities. Group III was exposed to a $20 \mathrm{db}$ attenuation tone present throughout the session. Intensity fades occurred in the signal at the same points in time in the session as did the fades accompanying intensity changes for Groups I and II. Group IV was run in the absence of any imposed sound intensity. For both control groups, reinforcements were programmed at the same points in the sessions as for Groups I and II.

\section{Results}

Each S's responses were partitioned in accordance with the sound stimulus program employed for Groups I and II. Whenever a particular intensity was scheduled, responses emitted during that interval accumulated on an associated counter. This response partitioning obtained for all groups.

The mean curve for each of the groups taken across 21 days of conditioning is shown in Fig. 1. The response data in Fig. 1A is plotted in terms of the number of responses emitted in each "intensity" category. Fig. 1B represents the same data in percent of total session responding. Despite the fact that no intensity changes were correlated with these categories in the Group III and IV conditions there is considerable correspondence among the four curves. This finding is supported by the results of a Lindquist (1953) Type VI analysis of variance carried out on the percent of total response data. The analysis yields no signficant differences among the four groups. However, when the four groups are combined, significant differences in response by intensity category emerge $(p<.001)$.

The data for Groups I and II shown in Fig. 1B was combined, yielding a mean curve of percentage of total response as a function of applied stimulus for eight animals. A line was fitted to these mean data points by the method of least squares, $(\mathrm{Y}=-0.062 \mathrm{X}+11.4)$. When an analysis of variance for related samples was performed (Lewis, 1960), it was found that the hypothesis 


\section{MEAN DISTRIBUTION OF RESPONSE (21 DAYS)}

A

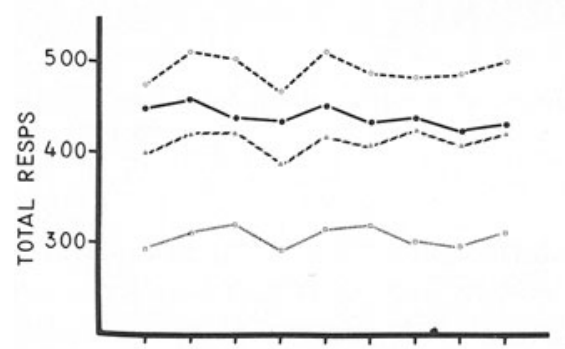

B

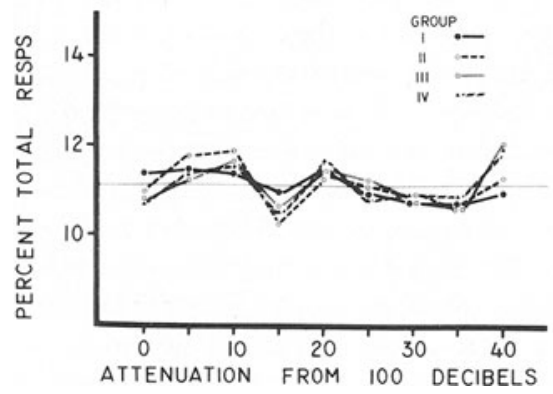

Fig. 1. Distribution of mean number and percent of total responses partitioned by intensity category.

of zero slope could not be rejected $(F<1.0)$. The dashed horizontal line in Fig. 1B represents $11.1 \%$ of total responding at each intensity. The data points would lie along this line if responding were equally distributed across intensities.

\section{Discussion}

Since the response distributions for the various groups do not differ from one another, the presence or absence of the imposed sound or fades would appear to be irrelevant to the response patterning during the session. There appears to be some stability in the response levels between groups when responding is partitioned into the nine categories which constitute the various intensity presentations in Groups I and II. These differences must be attributable to the point in session at which response rate is taken. Reinforcement availability was identical at all points in time for all groups, therefore it is not possible to parcel out the individual effects, if any, of point in time in session and probability of reinforcement. However, one or both of these factors, rather than any stimulus intensity preference, appears to be controlling the mementary response rate.

Although previous investigators have demonstrated both escape and avoidance behavior, employing sound levels roughly comparable to those used in this study, we noted no aversive effects. In addition to the different operations employed, perhaps another reason for this may lie in the fact that the demonstrably "noxious" sound stimuli were either white noise or a rather broad band of frequencies $(500-20 \mathrm{~K} \mathrm{cps})$, whereas the stimuli used in the present study were pure tones of a single frequency.

There was also a failure to demonstrate Hull's stimulus intensity dynamism effect. Although Hull did not make this point, it could be argued that since in this study there was no differential reinforcement as a function of intensity, the sound stimuli may have served no discriminative stimulus function. For a dynamism effect to be observed, it is suggested that a stimulus must become an $\mathrm{S}^{\mathrm{D}}$.

\section{References}

AZRIN, N. H. Some effects of noise on human behavior. J. exp. Anal. Behav., 1958, 1, 183-199.

BARRY. J. J. JR., \& HARRISON, J. M. Relation between stimulus intensity and strength of escape responding. Psychol. Rep., 1957, $3,3-8$.

BLOUGH, D. S. Generalization and preference on a stimulus-intensity continuum. J. exp. Anal. Behav., 1959, 2, 307-317.

CAMPBELL, B. A. The fractional reduction in noxious stimulation required to produce "just noticeable" learning. J. comp. physiol. Psychol., 1955, 43, 141-148.

HARRISON, J. M., \& ABELSON, R. M. The maintenance of behavior by the termination and onset of intense noise.J. exp. Anal. Behav., $1959,2,23 \sim 42$.

HULL, C. L. Stimulus intensity dynamism (V) and stimulus generalization. Psychol. Rev., 1949, 56, 67-76.

LEWIS, D. Quantitative methods in psychology. New York: McGrawHill, 1960.

LINDQUIST, E. F. Design and analysis of experiments in psychology and education. Boston: Houghton-Mifflin, 1953.

PIERREL, R., \& SHERMAN, J. G. Generalization of auditory intensity following discrimination training. J. exp. Anal. Behav., 1960, 3, 313-322.

Notes

1. This study was conducted as part of a research program under United States Public Health Service Grant HD-00928. A more detailed description of this study can be obtained from the senior author. 2. Mr. Scot Blue assisted the authors in the conduct of the experiment. 